\title{
CRITICAL BEHAVIORS AND CRITICAL VALUES OF BRANCHING RANDOM WALKS ON MULTIGRAPHS
}

\author{
DANIELA BERTACCHI, ${ }^{*}$ Università di Milano - Bicocca \\ FABIO ZUCCA, ${ }^{* *}$ Politecnico di Milano
}

\begin{abstract}
We consider weak and strong survival for branching random walks on multigraphs with bounded degree. We prove that, at the strong critical value, the process dies out locally almost surely. We relate the weak critical value to a geometric parameter of the multigraph. For a large class of multigraphs (which enlarges the class of quasi-transitive or regular graphs), we prove that, at the weak critical value, the process dies out globally almost surely. Moreover, for the same class, we prove that the existence of a pure weak phase is equivalent to nonamenability. The results are extended to branching random walks on weighted graphs.
\end{abstract}

Keywords: Branching random walk; phase transition; multigraph; amenability; tree

2000 Mathematics Subject Classification: Primary 60K35

\section{Introduction}

In recent years much study has been devoted to various stochastic processes, such as percolation, the Ising model, contact processes, and branching random walks, on general graphs (see, for instance, [3], [9], [10], [13], and [14], and see [11] for more references). A double motivation underlies the search for settings other than the usual $\mathbb{Z}^{d}$ : on one hand, the need for structures which may serve as models for inhomogeneous crystals, biological structures, or social networks and, on the other hand, the fact that on general graphs interesting phenomena, which are absent in $\mathbb{Z}^{d}$, are observed. In particular, the branching random walk (BRW) has been studied on trees (see [5], [7], [8], [12], and [14]) and on quasi-transitive graphs (see [15]).

In this paper we study the BRW on a connected multigraph $X$ with bounded degree (see Subsection 2.1 for the formal definition). Roughly speaking, a $\lambda$-BRW can be described by the following rules: each particle dies after an exponential time with parameter 1 and breeds independently on each edge at exponential intervals with parameter $\lambda$. We start with a finite number of particles; hence, the $\lambda$-BRW can be viewed as a continuous-time random walk on the countable state space of finite configurations $\eta \in \mathbb{N}^{X}$. On each site $x \in X$ the transitions are

$$
\eta(x) \rightarrow \eta(x)-1 \quad \text { at rate } \eta(x), \quad \eta(x) \rightarrow \eta(x)+1 \quad \text { at rate } \lambda \sum_{y \in X} n_{y x} \eta(y),
$$

where $\eta(x)$ is the number of particles at site $x$ and $n_{y x}$ is the number of edges from $y$ to $x$.

Received 21 December 2007; revision received 7 April 2008.

* Postal address: Dipartimento di Matematica e Applicazioni, Università di Milano - Bicocca, via Cozzi 53, 20125 Milano, Italy. Email address: daniela.bertacchi@unimib.it

** Postal address: Dipartimento di Matematica, Politecnico di Milano, piazza Leonardo da Vinci 32, 20133 Milano, Italy. Email address: fabio.zucca@polimi.it 
The BRW was originally introduced as a model for biological population dynamics (although it has been argued that this model is far from being satisfactory (see, for instance, the discussion in [6])) and, besides being interesting in itself, has also been studied for its relationship with the contact process (the process which has the same transition rules of the BRW but state space $\left.\{0,1\}^{X}\right)$. Indeed, the BRW stochastically dominates the contact process and has the property that the sum of two $\lambda$-BRWs is still a $\lambda$-BRW.

The $\lambda$-BRW on $\mathbb{Z}^{d}$ shows only two possible behaviors (called phases): if $\lambda \leq 1 / 2 d$, there is extinction almost surely; if $\lambda>1 / 2 d$, for all $t_{0}>0$, we have $\mathrm{P}\left(\eta_{t}(0)>0\right.$ for some $\left.t \geq t_{0}\right)>0$, where $\eta_{t}(0)$ is the number of particles at 0 at time $t$. The main interest in studying the BRW on trees is that a third phase appears. Indeed, we may identify the following two kinds of survival.

(i) Weak (or global) survival: the total number of particles is positive at each time.

(ii) Strong (or local) survival: the number of particles at one site $x$ is not eventually 0 .

Let us denote by $\lambda_{\mathrm{w}}$ and $\lambda_{\mathrm{s}}$ the infimum of the values $\lambda$ such that there is weak and strong survival, respectively, with positive probability. Clearly, $\lambda_{\mathrm{w}} \leq \lambda_{\mathrm{s}}$ and we may have three distinct phases corresponding to the following intervals for $\lambda$ : $\left[0, \lambda_{\mathrm{W}}\right),\left(\lambda_{\mathrm{w}}, \lambda_{\mathrm{s}}\right)$, and $\left(\lambda_{\mathrm{s}},+\infty\right)$. The middle interval may be empty; if, on the contrary, $\lambda_{\mathrm{w}}<\lambda_{\mathrm{s}}$ then we say that the BRW has a pure weak phase. In this phase the process leaves any finite subset eventually almost surely; hence, it survives globally by drifting to infinity (see [5] for details on the convergence to the boundary in the case of homogeneous trees).

This paper is devoted to three main issues: the identification of the critical value $\lambda_{\mathrm{w}}$, the behavior of the process at the critical values $\lambda=\lambda_{\mathrm{s}}$ and $\lambda=\lambda_{\mathrm{w}}$, and the existence of the pure weak phase. In [14] it was proved that $\lambda_{\mathrm{s}}$ is related to a particular asymptotic degree of the graph. We extend this characterization to multigraphs (Theorem 3.1) and we relate $\lambda_{\mathrm{W}}$ to another asymptotic degree (Theorems 3.2, 3.3, and 3.4). Moreover, we prove, by using generating function techniques, that if $\lambda=\lambda_{\mathrm{s}}$ then the process dies out locally almost surely and that, if $\lambda=\lambda_{\mathrm{w}}$, on a large class of multigraphs the process dies out globally almost surely (Theorem 3.5). The use of multigraphs is natural; indeed, given a vertex on a graph, the birth rate is equal for all neighbors, while on a multigraph we can consider more inhomogeneous situations (for a generalization, see Subsection 4.2). Moreover, some of our results need the requirement that in some sense 'there are only a finite number of types of vertices' (see Definition 3.1). This requirement, on the one hand, is naturally written by means of multigraphs and, on the other hand, it identifies our main class of multigraphs which includes both quasi-transitive and regular graphs.

As for conditions for the existence of the pure weak phase, we are led to investigate nonamenable graphs. Indeed, usually, nonamenable graphs are graphs where certain phenomena, absent in the amenable case, appear (see [11] for a survey). Nevertheless, a statement like 'nonamenability of the graph is equivalent to the existence of a pure weak phase for the BRW' has been disproved in [14]. The authors showed a nonamenable tree where the BRW has no pure weak phase and an amenable tree where there is such a phase (note that these counterexamples are both of bounded degree). Hence, we hope to prove a similar statement for a more restricted class of graphs. Work in this direction has been carried out in [15, Theorem 3.1], which states the equivalence between nonamenability and the existence of a pure weak phase for quasi-transitive graphs. We prove the same equivalence for our main class of multigraphs (Theorem 3.6 and Example 3.3). 
Let us give the outline of the paper. In Section 2 we introduce the main definitions and we define some generating functions and a generalized branching process which will be useful in the sequel. Moreover, we introduce two asymptotic degrees, $M_{\mathrm{s}}$ and $M_{\mathrm{w}}$, which depend only on the geometric structure of the multigraph.

Subsection 3.1 is devoted to the detailed study of $\lambda_{\mathrm{s}}$ and $\lambda_{\mathrm{w}}$. We give a sufficient condition for the absence of the pure weak phase, which, in particular, implies that there is no weak phase on subexponentially growing multigraphs (Remark 3.1). We prove that on multigraphs $\lambda_{\mathrm{s}}=1 / M_{\mathrm{s}}$ (Theorem 3.1), and we show that, for a large class of multigraphs, $\lambda_{\mathrm{w}}=1 / M_{\mathrm{w}}$ (Theorem 3.2). Clearly, for this class, we have $\lambda_{\mathrm{w}}<\lambda_{\mathrm{s}}$ if and only if $M_{\mathrm{s}}<M_{\mathrm{w}}$. We give two different sufficient conditions for a multigraph to satisfy the hypothesis of Theorem 3.2. The first condition (Theorem 3.3) is satisfied, for instance, by certain radial trees which are not quasitransitive; for these trees, we show that nonamenability is equivalent to the existence of the pure weak phase (Example 3.3). As for the second condition (Theorem 3.4), we introduce a class of morphisms (Definition 3.1) of multigraphs and we show that it preserves $\lambda_{\mathrm{w}}, M_{\mathrm{w}}$, and, in some cases, $\lambda_{\mathrm{s}}$ (Proposition 3.1). By using these morphisms, the class of $\mathcal{F}$-multigraphs is defined; this class satisfies the hypothesis of Theorem 3.2. In Subsection 3.2 we prove that on general multigraphs the $\lambda_{\mathrm{s}}$-BRW dies out locally almost surely and that on $\mathcal{F}$-multigraphs the $\lambda_{\mathrm{w}}$-BRW dies out globally almost surely (Theorem 3.5). The main result of Subsection 3.3 (Theorem 3.6) along with Theorem 3.4 yields, for nonoriented $\mathcal{F}$-multigraphs, the equivalence of the following conditions: (i) $\lambda_{\mathrm{w}}<\lambda_{\mathrm{s}}$, (ii) $M_{\mathrm{s}}<M_{\mathrm{w}}$, and (iii) nonamenability. In Subsection 3.4 some examples of multigraphs, which can be studied via our results, are given.

The BRW studied in Section 3 may be viewed as a population which reproduces following an 'edge breeding' pattern, while some authors prefer a 'site breeding' pattern. In Section 4 we consider this modification of the BRW. These two versions of the BRW are essentially equivalent on regular graphs, while in the general setting the behavior of the 'site breeding' BRW can be much more easily characterized (Theorem 4.1). We show that BRWs and modified BRWs may both be seen as particular cases of BRWs on weighted graphs. Most of the results given in the previous sections still hold in this general setting.

Section 5 is devoted to a final discussion of open questions.

\section{Basic definitions and preliminaries}

\subsection{Multigraphs}

A countable (or finite) multigraph is a couple $(X, E(X))$, where $X$ is the countable (or finite) set of vertices and $E(X) \subseteq X \times X \times \mathbb{N}_{*}$ is the set of (oriented) edges (where $\mathbb{N}_{*}$ is the set of positive natural numbers); we define the number of edges from $x$ to $y$ as $n_{x y}:=$ $|\{i:(x, y, i) \in E(X)\}| \equiv \max \{i:(x, y, i) \in E(X)\}$ (where $|\cdot|$ denotes cardinality). We denote by $D(x):=\left\{y \in X: n_{x y}>0\right\}$ the set of neighbors of $x$ and by $\operatorname{deg}(x):=\sum_{y \in D(x)} n_{x y}$ the degree of $x$. If $n_{x y}=n_{y x}$ for all $x, y \in X$ then the multigraph is called nonoriented. A multigraph is a graph if and only if $n_{x y}=\mathbf{1}_{D(x)}(y)$,

A path from $x$ to $y$ of length $n$ is a couple of sequences

$$
\left(\left\{x=x_{0}, x_{1}, \ldots, x_{n}=y\right\},\left\{k_{1}, \ldots, k_{n}\right\}\right)
$$

such that $n_{x_{i} x_{i+1}} \geq k_{i+1}>0$ for all $i=0,1, \ldots, n-1$. The multigraph is said to be connected if there exists a path (of suitable length) from $x$ to $y$ for all $x, y \in X$. From now on, the multigraph will always be connected and of bounded degree, that is, $M(X):=\sup _{x \in X} \operatorname{deg}(x)<+\infty$; obviously, $M$ depends on $(X, E(X))$, but to avoid cumbersome notation, the dependence on 
the set of edges will be tacitly understood. The same implicit assumption will be made for all quantities depending on the multigraph. Moreover, if not explicitly stated, the multigraph does not need to be nonoriented.

Let $\gamma_{x, y}^{n}$ be the number of paths of length $n$ from $x$ to $y$ (and $\gamma_{x, y}^{0}:=\delta_{x, y}$ ). More explicitly, to each sequence $\left\{x=x_{0}, x_{1}, \ldots, x_{n}=y\right\}$ there corresponds a set of $\prod_{i=0}^{n-1} n_{x_{i} x_{i+1}}$ paths in the multigraph, whence $\gamma_{x, y}^{n}$ is the sum over all the sequences $\left\{x=x_{0}, x_{1}, \ldots, x_{n}=y\right\}$ of $\prod_{i=0}^{n-1} n_{x_{i} x_{i+1}}$. Moreover, let $T_{x}^{n}$ be the number of paths from $x$ of length $n$, that is, $T_{x}^{n}:=\sum_{y \in X} \gamma_{x, y}^{n}$. Finally, let $\phi_{x, y}^{n}$ be the number of paths of length $n$ starting from $x$ and reaching $y$ for the first time; to be precise, $\phi_{x, y}^{n}$ is the number of paths $\left(\left\{x=x_{0}, x_{1}, \ldots, x_{n}=y\right\}\right.$, $\left.\left\{k_{1}, \ldots, k_{n}\right\}\right)$ such that $x_{i} \neq y$ for all $i=1, \ldots, n-1$. By definition, $\phi_{x, y}^{0}:=0$ for all $x, y \in X$.

For $\gamma_{x, y}^{n}$ and $T_{x}^{n}$, the following recursive relations hold for all $n, m \geq 0$ :

$$
\begin{aligned}
\gamma_{x, y}^{n+m} & =\sum_{w \in X} \gamma_{x, w}^{n} \gamma_{w, y}^{m}, & & \gamma_{x, y}^{1}=n_{x y}, \\
T_{x}^{n+m} & =\sum_{w \in X} \gamma_{x, w}^{m} T_{w}^{n}, & & T_{x}^{1}=\operatorname{deg}(x),
\end{aligned}
$$

and, for all $n \geq 1$,

$$
\gamma_{x, y}^{n}=\sum_{i=0}^{n} \phi_{x, y}^{i} \gamma_{y, y}^{n-i}
$$

Given any vertex $x \in X$ and $n \in \mathbb{N}$, we define $\rho(x, y):=\min \left\{i: \gamma_{x, y}^{i}>0\right\}$ and $B(x, n):=$ $\{y \in X: \rho(x, y) \leq n\}$; note that $\rho$ is a metric if $n_{x y}>0$ is equivalent to $n_{y x}>0$ for all $x, y \in X$ (for instance, in the case of nonoriented multigraphs).

By using the number of paths, it is possible to introduce two asymptotic degrees, namely

$$
M_{\mathrm{S}}(X):=\limsup _{n}\left(\gamma_{x, y}^{n}\right)^{1 / n}, \quad M_{\mathrm{W}}(X):=\limsup _{n}\left(T_{x}^{n}\right)^{1 / n} .
$$

It is easy to show that the above definitions do not depend on the choice of $x, y \in X$ and simple arguments of supermultiplicativity show that $M_{\mathrm{S}}(X)=\lim _{n \rightarrow \infty}\left(\gamma_{x, x}^{d n}\right)^{1 / d n}=\sup _{n}\left(\gamma_{x, x}^{d n}\right)^{1 / d n}$, where $d:=\operatorname{gcd}\left\{n: \gamma_{x, x}^{n}>0\right\}$ is the period of the multigraph (which does not depend on the choice of $x$ ). Analogously, $M_{\mathrm{S}}(X)=\lim _{n \rightarrow \infty}\left(\gamma_{x, y}^{d n+i}\right)^{1 /(d n+i)}$, where $0 \leq i \leq d-1$ is uniquely chosen such that $\gamma_{x, y}^{n}>0$ implies that $n=i(\bmod d)$. In the rest of the paper, whenever there is no ambiguity, we will denote $M(X), M_{\mathrm{s}}(X)$, and $M_{\mathrm{w}}(X)$ simply by $M, M_{\mathrm{s}}$, and $M_{\mathrm{w}}$.

By definition, $1 \leq M_{\mathrm{S}} \leq M_{\mathrm{w}} \leq M$. We note that $M_{\mathrm{w}}=M$ if the multigraph is regular, that is, it has constant degree. It is well known that, for a regular nonoriented graph, $M_{\mathrm{S}}<M_{\mathrm{w}}$ if and only if it is nonamenable (see Subsection 3.3 for the definition). A simple case where $M_{\mathrm{W}}=M_{\mathrm{S}}$ is provided by the following proposition.

Proposition 2.1. Let $(X, E(X))$ be a nonoriented multigraph. If $|B(x, n)|^{1 / n} \rightarrow 1$ for some (equivalently, for all) $x \in X$ then $M_{\mathrm{w}}=M_{\mathrm{S}}$.

Proof. It is enough to prove that $M_{\mathrm{w}} \leq M_{\mathrm{s}}$. Note that, by the Cauchy-Schwarz inequality,

$$
M_{\mathrm{s}}^{2 n} \geq \gamma_{x, x}^{2 n}=\sum_{y \in X} \gamma_{x, y}^{n} \gamma_{y, x}^{n}=\sum_{y \in B(x, n)}\left(\gamma_{x, y}^{n}\right)^{2} \geq \frac{\left(\sum_{y} \gamma_{x, y}^{n}\right)^{2}}{|B(x, n)|}=\frac{\left(T_{x}^{n}\right)^{2}}{|B(x, n)|}
$$

hence,

$$
M_{\mathrm{S}} \geq \limsup _{n} \sqrt[2 n]{\frac{\left(T_{x}^{n}\right)^{2}}{|B(x, n)|}}=\limsup _{n} \sqrt[n]{T_{x}^{n}}=M_{\mathrm{w}}
$$




\subsection{Generating functions}

In order to find some characterizations of $M_{\mathrm{s}}$ and $M_{\mathrm{w}}$, let us define the generating functions

$$
H(x, y \mid \lambda):=\sum_{n=0}^{\infty} \gamma_{x, y}^{n} \lambda^{n}, \quad \Theta(x \mid \lambda):=\sum_{n=0}^{\infty} T_{x}^{n} \lambda^{n},
$$

with radius of convergence $1 / M_{\mathrm{S}}$ and $1 / M_{\mathrm{W}}$, respectively. Of course, for all $\lambda \in \mathbb{C}$ such that $|\lambda|<1 / M_{\mathrm{w}}$, we have $\Theta(x \mid \lambda)=\sum_{y \in Y} H(x, y \mid \lambda)$ and the following relations hold:

$$
\begin{aligned}
& H(x, y \mid \lambda)=\delta_{x, y}+\lambda \sum_{w \in X} \gamma_{x, w}^{1} H(w, y \mid \lambda) \\
&=\delta_{x, y}+\lambda \sum_{w \in X} H(x, w \mid \lambda) \gamma_{w, y}^{1} \quad \text { for all } \lambda \in \mathbb{C} \text { such that }|\lambda|<\frac{1}{M_{\mathrm{s}}}, \\
& \Theta(x \mid \lambda)=1+\lambda \sum_{w \in X} \gamma_{x, w}^{1} \Theta(w \mid \lambda) \quad \text { for all } \lambda \in \mathbb{C} \text { such that }|\lambda|<\frac{1}{M_{\mathrm{w}}}
\end{aligned}
$$

We define

$$
\Phi(x, y \mid \lambda):=\sum_{n=1}^{\infty} \phi_{x, y}^{n} \lambda^{n}
$$

it is easy to see that

$$
\Phi(x, x \mid \lambda)=\lambda \sum_{y \in X, y \neq x} \gamma_{x, y}^{1} \Phi(y, x \mid \lambda)+\lambda \gamma_{x, x}^{1},
$$

and if $x, y, w \in X$ are distinct vertices such that every path from $x$ to $y$ contains $w$ then $\Phi(x, y \mid \lambda)=\Phi(x, w \mid \lambda) \Phi(w, y \mid \lambda)$. Moreover,

$$
H(x, y \mid \lambda)=\Phi(x, y \mid \lambda) H(y, y \mid \lambda)+\delta_{x, y} \text { for all } \lambda \text { such that }|\lambda|<1 / M_{\mathrm{s}} .
$$

Since the radius of the series $H(x, x \mid \cdot)$ does not depend on the choice of $x \in X$ and since

$$
H(x, x \mid \lambda)=\frac{1}{1-\Phi(x, x \mid \lambda)} \quad \text { for all } \lambda \in \mathbb{C} \text { such that }|\lambda|<\frac{1}{M_{\mathrm{S}}},
$$

we have $1 / M_{\mathrm{S}}=\max \{\lambda \geq 0: \Phi(x, x \mid \lambda) \leq 1\}$ for all $x \in X$ (remember that $\Phi(x, x \mid \cdot)$ is left continuous on $\left[0,1 / M_{\mathrm{S}}\right]$ and that $1 /(1-\Phi(x, x \mid \lambda))$ has no analytic prolongation in $\left.1 / M_{\mathrm{S}}\right)$.

The computation of $M_{\mathrm{w}}$ is not easy in general, but in the case of finite multigraphs there is a simple characterization of $M_{\mathrm{w}}$. In the following proposition, $I$ is the identity matrix.

Proposition 2.2. Let $(X, E(X))$ be an irreducible, finite multigraph with adjacency matrix $N:=\left(n_{x y}\right)_{x, y \in X}$. Then

$$
\frac{1}{M_{\mathrm{w}}}=\frac{1}{M_{\mathrm{s}}}=\min \{\lambda>0: \operatorname{det}(\lambda N-I)=0\} .
$$

Proof. We use the same notation $N$ for the matrix and the linear operator. By the PerronFrobenius theorem, there exists an eigenvalue $\sigma_{0}>0$ of $N$ such that any other eigenvalue $\sigma$ satisfies $|\sigma|<\sigma_{0}$, and the same holds for $N^{\top}$. Moreover, $\operatorname{dim}\left(\operatorname{Ker}\left(N^{\top}-\sigma_{0} I\right)\right)=1$ and it is possible to choose the eigenvector $v$ in such a way that $v>0$. It is clear that any vector $w<0$ 
cannot possibly belong to $\operatorname{Rg}\left(N-\sigma_{0} I\right) \equiv \operatorname{Ker}\left(N^{\top}-\sigma_{0} I\right)^{\perp}$ since $\langle w, v\rangle<0$. Then (2.1) (which holds for $|\lambda|<1 / M_{\mathrm{w}}$ ) can be written as

$$
(\lambda N-I) \Theta(\lambda)=-\left(\begin{array}{c}
1 \\
1 \\
\vdots \\
1
\end{array}\right),
$$

and has no solutions if $\lambda=1 / \sigma_{0}$. On the other hand, (2.3) defines a holomorphic (vector) function $\Theta^{\prime}(\lambda)=-(\lambda N-I)^{-1}(1,1, \ldots, 1)^{\top}$ on $\left\{\lambda \in \mathbb{C}:|\lambda|<1 / \sigma_{0}\right\}$. Note that $\Theta^{\prime}$ coincides with $\Theta$ on $\left\{\lambda \in \mathbb{C}:|\lambda|<\min \left\{1 / \sigma_{0}, 1 / M_{\mathrm{w}}\right\}\right\}$; hence, $1 / \sigma_{0} \leq 1 / M_{\mathrm{w}}$. If $1 / \sigma_{0}<1 / M_{\mathrm{w}}$ then there would be an analytic prolongation of $\Theta^{\prime}$ to $1 / \sigma_{0}$ and, by continuity, (2.3) would hold for $\lambda=1 / \sigma_{0}$.

\subsection{Generalized branching process}

In the classical branching process (see, for instance, [4, Chapter 1]) there is a unique offspring distribution according to which each individual breeds. We consider a generalized branching process where each father may have different types of children and each of them breeds according to a specific distribution which depends on its type and on the father. To be more specific, let $\mathcal{T}=\left(\bigcup_{i=0}^{\infty} \mathbb{N}_{*}^{2 i}, E(\mathcal{T})\right)$, where $\mathbb{N}_{*}^{0}:=\{o\}$ and $o$ is the root of the tree $\mathcal{T}$. Identifying as usual $\mathbb{N}_{*}^{2 n} \times \mathbb{N}_{*}^{2}$ with $\mathbb{N}_{*}^{2 n+2}$, the set of edges is

$$
E(\mathcal{T}):=\left\{(x, y) \in \mathcal{T}: \text { there exists } k \in \mathbb{N}_{*}^{2}, y=(x, k)\right\} \cup\left\{(o, k): k \in \mathbb{N}_{*}^{2}\right\} .
$$

Roughly speaking, $y=(x, i, j)$ means that $y$ is the $j$ th son of type $i$ of its father $x$ (whereas $(i, j)$ is the $j$ th son of type $i$ of $o$ ), and the oriented edges are drawn from fathers to sons. Moreover, $\bigcup_{i=0}^{n} \mathbb{N}_{*}^{2 i}$ represents the genealogic tree of the progeny of $o$ up to the $n$th generation. We provide each individual $x$ with a distribution $\mu_{x}$ such that if $x=(v, i, j)$ and $y=(v, i, k)$ then $\mu_{x} \equiv \mu_{y}$ (that is, the offspring distribution depends only on the father and on the type). Now, each distribution is defined on the countable space $\mathcal{E}:=\left\{f \in \mathbb{N}^{\mathbb{N}}: S(f)<+\infty\right\}$, where $S(f)=\sum_{i=1}^{\infty} f(i)$. To be more precise, it is possible to construct a canonical probability space $(\Omega, \mathcal{A}, \mathrm{P})$ supporting the generalized branching process and such that $\mathrm{P}$ satisfies

$$
\mu_{x}(f)=\mathrm{P}\left(\bigcap_{i=1}^{\infty}\{x \text { has } f(i) \text { sons of type } i\}\right) \text { for all } f \in \mathcal{E} .
$$

Moreover, for every $x \in \mathcal{T}$, let $v_{x}$ be the distribution of the total number of children of $x$, that is, $v_{x}(k)=\mu_{x}(\{f: S(f)=k\})$ for all $k \in \mathbb{N}$. Take a family of independent $\mathcal{E}$-valued random variables $\left\{Z_{x}\right\}_{x \in \mathcal{T}}$ such that $Z_{x}$ has distribution $\mu_{x}$.

Let us recursively construct this generalized branching process $\left\{B_{n}\right\}_{n \geq 0}$ :

$$
B_{0}=\{o\}, \quad B_{n+1}=\left\{(v, i, j): v \in B_{n}, 1 \leq j \leq Z_{v}(i)\right\},
$$

where $B_{n}$ is the $n$th generation and its member $v$ has exactly $Z_{v}(i)$ children of type $i$. Extinction is the event ( $B_{n}=\varnothing$ eventually).

Lemma 2.1. Let $G_{x}(z)$ be the generating function of $v_{x}$, and suppose that there exists $\delta \in[0,1)$ such that $G_{x}(\delta) \leq \delta$ for all $x \in \mathcal{T}$. Then $\mathrm{P}\left(B_{n}=\varnothing\right.$ eventually $) \leq \delta$. 
Proof. Denote by $A_{n}^{x}$ the event of extinction before the $n$th generation of the progeny of $x$. Let $q_{n}^{x}:=\mathrm{P}\left(A_{n}^{x}\right)$. Clearly, $q_{n}^{x}$ depends only on the father and the type of $x$; we claim that $q_{n}^{x} \leq \delta$ for all $x \in \mathcal{T}$. We proceed by induction on $n$. Obviously, for each $x \in \mathcal{T}$, $q_{0}^{x}=v_{x}(0)=G_{x}(0) \leq \delta$. By induction, using the hypothesis of independence,

$$
\begin{aligned}
q_{n+1}^{x} & =\mathrm{P}\left(\bigcup_{i=0}^{\infty} \bigcup_{f: S(f)=i} \bigcap_{j=1}^{\infty} \bigcap_{k=1}^{f(j)} A_{n}^{(x, j, k)}\right) \\
& =\sum_{i=0}^{\infty} \sum_{f: S(f)=i} \mu_{x}(f) \prod_{j=1}^{\infty}\left(q_{n}^{(x, j, 1)}\right)^{f(j)} \\
& \leq \sum_{i=0}^{\infty} \sum_{f: S(f)=i} \mu_{x}(f) \delta^{S(f)} \\
& =\sum_{i=0}^{\infty} \delta^{i} \sum_{f: S(f)=i} \mu_{x}(f) \\
& =\sum_{i=0}^{\infty} \delta^{i} v_{x}(i) \\
& =G_{x}(\delta) \\
& \leq \delta
\end{aligned}
$$

Now, $q_{n}^{o} \uparrow \mathrm{P}\left(B_{n}=\varnothing\right.$ eventually) and $\delta \geq \lim _{n} q_{n}^{o}$. This completes the proof.

This lemma trivially applies when each distribution $\mu_{x}$ is drawn from a finite set of distributions such that the corresponding $v_{x}$ represents a supercritical branching process. In this case we have a finite number of fixed points in $[0,1)$ for the generating functions and $\delta$ may be taken as the maximum among them (indeed this is what we do in Theorem 3.2, below).

\section{Main results}

\subsection{The critical values}

The critical values $\lambda_{\mathrm{s}}$ and $\lambda_{\mathrm{w}}$ separate different behaviors of the $\lambda$-BRW. Namely, given nonextinction, if $\lambda>\lambda_{\mathrm{s}}$ then the conditional expected value of the number of particles per site tends to $\infty$, while if $\lambda>\lambda_{\mathrm{w}}$ then the conditional probability that the total number of particles on the graph tends to $\infty$ is equal to 1 . Indeed, the BRW (starting with a finite number of particles) is a continuous-time random walk on the countable state space of finite configurations $\eta \in \mathbb{N}^{X}$, with a trap state in $\underline{0}$ (the configuration with no particles). Hence, all the states but $\underline{0}$ are transient and the process which does not hit $\underline{0}$ leaves $A_{k}=\left\{\eta \in \mathbb{N}^{X}: \sum_{x \in X} \eta(x) \leq k\right\}$ eventually for all $k \in \mathbb{N}$. Indeed, the probability of reaching $\underline{0}$ starting from any configuration in $A_{k}$ is bounded from below by a positive constant (the reproduction rate is bounded from above); hence, the claim follows.

Here we investigate the critical values $\lambda_{\mathrm{s}}$ and $\lambda_{\mathrm{w}}$ and their relationship with $M_{\mathrm{s}}$ and $M_{\mathrm{w}}$. Since the critical values do not depend on the number of particles at $t=0$ (nor on their location), we suppose that the initial state is one particle at a fixed vertex $o \in X$. To each particle $p$ (present at some time at a site $x$ ) there corresponds a (unique) reproduction trail, starting from the initial particle located at $o$ at time 0 , reconstructing the genealogy of $p$. 
Roughly speaking, the (space-time) reproduction trail corresponding to $p$ is a path $\left(\left\{x_{0}=o\right.\right.$, $\left.\left.x_{1}, \ldots, x_{n-1}, x_{n}=x\right\},\left\{k_{1}, \ldots, k_{n}\right\}\right)$ along with a sequence $\left(t_{0}, \ldots, t_{n-1}\right)$, where $t_{0}$ is the epoch when the original particle in $o$ generated the ancestor of $p$ in $x_{1}$ (through the edge $\left(o, x_{1}, k_{1}\right)$ ) and, for $i=1, \ldots, n-1, t_{i}$ is the epoch when the ancestor in $x_{i}$ generated the one in $x_{i+1}$ (through the edge $\left(x_{i}, x_{i+1}, k_{i+1}\right)$ ). Clearly, setting $t_{-1}=0$, then, for all $i=0, \ldots, n-1$, $t_{i}-t_{i-1}$ is the realization of an exponential random variable with rate $\lambda$ (it is tacitly understood that each ancestor is alive when breeding). Such a trail is said to have length $n$. For a detailed construction, we refer the reader to [14, Section 3] (where what we call a reproduction trail is an infection trail). Note that the expected number of trails along a path of length $n$ is $\lambda^{n}$; hence, to each sequence $\left\{x_{0}, x_{1}, \ldots, x_{n}\right\}$ there corresponds a number $\lambda^{n} \prod_{i=0}^{n-1} n_{x_{i} x_{i+1}}$ of expected trails.

In [14, Lemma 3.1] it was proved that $\lambda_{\mathrm{s}}=1 / M_{\mathrm{S}}$ for any graph. We use a different approach to extend this result to multigraphs; this approach allows us to study the critical behavior when $\lambda=\lambda_{\mathrm{s}}$ (see Theorem 3.5, below).

Theorem 3.1. For each multigraph $(X, E(X))$, we have $\lambda_{\mathrm{s}}=1 / M_{\mathrm{s}}$.

Proof. Let us consider a path $\Pi:=\left(\left\{o=x_{0}, x_{1}, \ldots, x_{n}=o\right\},\left\{k_{1}, \ldots, k_{n}\right\}\right)$ and let us define its number of cycles $\mathcal{L}(\Pi):=\left|\left\{i=1, \ldots, n: x_{i}=o\right\}\right|$; the expected number of trails along such a path is $\lambda^{n}$ (hence, to each sequence $\left\{x_{0}, x_{1}, \ldots, x_{n}\right\}$ there corresponds a number $\lambda^{n} \prod_{i=0}^{n-1} n_{x_{i} x_{i+1}}$ of expected trails). Disregarding the original time scale, to the BRW there corresponds a Galton-Watson branching process: given any particle $p$ in $o$ (corresponding to a trail with $n$ cycles), define its children as all the particles whose trail is a prolongation of the trail of $p$ and is associated with a spatial path with $n+1$ cycles. Hence, a particle is of the $k$ th generation if and only if the corresponding trail has $k$ cycles; moreover, it has one (and only one) parent in the $(k-1)$ th generation. Since each particle behaves independently of the others, the process is Markovian. Thus, the BRW survives if and only if this branching process does. The expected number of children of the branching process is the sum over $n$ of the expected number of trails of length $n$ and one cycle, that is, $\sum_{n=1}^{\infty} \phi_{o, o}^{n} \lambda^{n}=\Phi(o, o \mid \lambda)$. Thus, we have almost sure local extinction if and only if $\Phi(o, o \mid \lambda) \leq 1$, that is, $\lambda \leq 1 / M_{\mathrm{S}}$ (see (2.2) and the remark thereafter).

We now focus our attention on the weak critical value.

Lemma 3.1. For every multigraph, we have $\lambda_{\mathrm{w}} \geq 1 / M_{\mathrm{w}}$.

Proof. Since the average number of trails on a fixed path of length $n$ starting from $(o, 0)$ is $\lambda^{n}$, the average number of all the trails on any path from $(o, 0)$ is $\sum_{n=0}^{\infty} \lambda^{n} T_{o}^{n}$. If $\lambda M_{\mathrm{W}}<1$ then this sum is finite; hence, the number of reproduction trails is almost surely finite and there is no weak survival.

Remark 3.1. For every multigraph, if $M_{\mathrm{w}}=M_{\mathrm{s}}$, there is no pure weak survival (since $1 / M_{\mathrm{w}} \leq$ $\lambda_{\mathrm{w}} \leq \lambda_{\mathrm{s}}=1 / M_{\mathrm{s}}$ ). Examples are subexponentially growing graphs (recall Proposition 2.1) such as $\mathbb{Z}^{d}$ or $d$-dimensional combs (see [1] for the definition).

Let us now consider whether $\lambda_{\mathrm{w}}=1 / M_{\mathrm{w}}$. The following theorem states that this equality holds if the multigraph satisfies the following geometric condition:

$$
\text { for all } \varepsilon>0 \text {, there exists } \bar{n}=\bar{n}(\varepsilon) \text { such that } \sup _{n \leq \bar{n}} \sqrt[n]{T_{x}^{n}} \geq M_{\mathrm{w}}-\varepsilon \text { for all } x \in X \text {. }
$$

Note that, by definition of $M_{\mathrm{w}}$, for all fixed $\varepsilon>0$ and $x \in X$, there exists $n_{x}$ such that $\sqrt[n_{x}]{T_{x}^{n_{x}}} \geq M_{\mathrm{w}}-\varepsilon$. Condition (3.1) is a request of uniformity in $x$. 
Theorem 3.2. If $(X, E(X))$ is a multigraph such that condition (3.1) holds then $\lambda_{\mathrm{w}}=1 / M_{\mathrm{w}}$.

Proof. Fix $\varepsilon>0$ and $\lambda$ such that $\lambda\left(M_{\mathrm{w}}-\varepsilon\right)>1$. We associate to the BRW a generalized branching process where the type of each particle is the site where it is born (although in Subsection 2.3 the type was indexed by $\mathbb{N}$, this is not a restriction since $X$ is at most countable). For all $x \in X$, define $n_{x}$ to be the smallest positive integer such that $\sqrt[n_{x}]{T_{x}^{n_{x}}} \geq M_{\mathrm{w}}-\varepsilon$. In this generalized branching process the 'children' of the initial particle (which represents the root of the tree of the process) are all the particles associated with trails of length $n_{o}$ starting from $o$. Each of these trails ends on a specific vertex in $B\left(o, n_{o}\right)$, which represents the type of children generated there. The offspring distribution $\mu_{o}$ is supported on $\mathcal{E}_{o}:=\{f \in \mathcal{E}: f(x)=$ 0 for all $\left.x \notin B\left(o, n_{o}\right)\right\}$ and satisfies

$$
\mu_{o}(f)=\mathrm{P}\left(\bigcap_{y \in B\left(o, n_{o}\right)}\left\{K_{y}=f(y)\right\}\right) \text { for all } f \in \mathcal{E}_{o},
$$

where $\mathrm{P}$ is the probability on the space where the BRW is defined and $K_{y}$ is the (random) number of trails of length $n_{o}$ starting at $o$ and ending at $y$. The corresponding $v_{o}$ is supercritical in the sense that

$$
G_{o}^{\prime}(1) \equiv \sum_{n=0}^{\infty} n v_{o}(n)=\lambda^{n_{o}} T_{o}^{n_{o}}>1 .
$$

This means that $G_{o}$ has a fixed point $\delta_{o}<1$.

Analogously, we repeat this construction for any particle at any site $x$. The children of such a particle $p$ are the particles associated with trails which are prolongations of the trail of $p$, and the difference between the lengths of the prolongation and of the trail of $p$ is $n_{x}$. Clearly, the offspring distribution $\mu_{x}$ is supported on $\mathcal{E}_{x}:=\left\{f \in \mathcal{E}: f(z)=0\right.$ for all $\left.z \notin B\left(x, n_{x}\right)\right\}$ and is defined as

$$
\mu_{x}(f)=\mathrm{P}\left(\bigcap_{y \in B\left(x, n_{x}\right)}\left\{K_{y}=f(y)\right\}\right) \text { for all } f \in \mathcal{E}_{x},
$$

where $K_{y}$ is the (random) number of prolongations, ending at $y$, of the trail of $p$ such that the difference between the lengths of the prolongation and of the trail of $p$ is $n_{x}$. By the Markov property, these laws do not depend on the particle, but only on the site $x$; hence, the definition is well posed. More precisely, $\mu_{x}$ depends only on the submultigraph $B\left(x, n_{x}\right)$. We call $G_{x}$ the generating function of $v_{x}$.

The generating functions, $G_{x}$, are taken from a finite set of $G \mathrm{~s}$; indeed, in a bounded degree multigraph the set of the equivalence classes up to isometries of the balls of radius at most $\bar{n}$ is finite. Since all these generating functions are convex, we may apply Lemma 2.1 with $\delta=\max \left\{\delta_{x}: x \in X\right\}$, finding that the generalized branching process is supercritical. Since, for each $x \in X$, we consider only the particles generated along a path of length $n_{x}$ (starting from $x$ ), the generalized branching process is dominated by the total number of particles of the original BRW; hence, this last one is supercritical as well. Since $\varepsilon$ was arbitrary, we deduce that $\lambda_{\mathrm{w}} \leq 1 / M_{\mathrm{w}}$. Lemma 3.1 yields the conclusion.

A large family of multigraphs for which condition (3.1) holds is described by the following theorem. Roughly speaking, the geometrical request therein is a sort of 'translational quasiinvariance' which allows us to compare all $T_{x}^{n}$ with a single $T_{x_{0}}^{n}$. 
Theorem 3.3. Let $(X, E(X))$ be a multigraph; let us suppose that there exists $x_{0} \in X, Y \subseteq X$, and $n_{0} \in \mathbb{N}$ such that

(a) for all $x \in X$, we have $B\left(x, n_{0}\right) \cap Y \neq \varnothing$,

(b) for all $y \in Y$, there exists an injective map $\varphi_{y}: X \rightarrow X$ such that $\varphi_{y}\left(x_{0}\right)=y$ and $n_{\varphi_{y}(x) \varphi_{y}(z)} \geq n_{x z}$ for all $x, z \in X$.

Then condition (3.1) holds and $\lambda_{\mathrm{w}}=1 / M_{\mathrm{w}}$.

Proof. We fix $\varepsilon>0$. For any given $x \in X$, condition (a) implies the existence of $y \in Y$ such that $\rho(x, y) \leq n_{0}$; hence, $T_{y}^{n} \leq T_{x}^{n+n_{0}}$ for all $n \in \mathbb{N}$. Using condition (b), we have $T_{y}^{n} \geq T_{x_{0}}^{n}$ for all $n \in \mathbb{N}$, which in turn implies that $T_{x}^{n+n_{0}} \geq T_{x_{0}}^{n}$. Since $\lim \sup _{n \rightarrow \infty}\left(T_{x_{0}}^{n}\right)^{1 /\left(n+n_{0}\right)^{y}}=M_{\mathrm{w}}$, we may find $n_{1} \in \mathbb{N}$ such that $\left(T_{x_{0}}^{n_{1}}\right)^{1 /\left(n_{1}+n_{0}\right)} \geq M_{\mathrm{w}}-\varepsilon$, whence $\bar{n}(\varepsilon):=n_{1}+n_{0}$ satisfies the hypothesis of Theorem 3.2.

For a nontrivial example of trees satisfying the hypotheses of the previous theorem, see Example 3.3, below. Another important class of multigraphs satisfying condition (3.1) is described by the following definition (see also Theorem 3.4, below).

Definition 3.1. Let $(X, E(X))$ and $(Y, E(Y))$ be two multigraphs. A map $\varphi: X \rightarrow Y$ is called a local isomorphism from $X$ onto $Y$ if and only if, for all $x \in X$ and $y \in Y$, we have $\sum_{z \in X: \varphi(z)=y} n_{x z}^{X}=n_{\varphi(x) y}^{Y}$.

We say that a multigraph $(X, E(X))$ is an $\mathcal{F}$-multigraph if it is locally isomorphic to a finite multigraph. We say that a graph $(X, E(X))$ is an $\mathcal{F}$-graph if it is locally isomorphic to a finite graph.

Note that a local isomorphism from $X$ to $Y$ does not imply the existence of a local isomorphism from $Y$ to $X$. Moreover, it is easy to show that, for any local isomorphism, $\varphi\left(D_{X}(x)\right)=D_{Y}(\varphi(x))$ and that, for all $n \in \mathbb{N}$ and for all $x, y \in X$,

$$
\sum_{z \in X: \varphi(z)=y} \gamma_{x, z}^{n}=\tilde{\gamma}_{\varphi(x), y}^{n}, \quad T_{x}^{n}(X)=T_{\varphi(x)}^{n}(Y),
$$

where $\tilde{\gamma}$ refers to paths in $Y$. The second equation in (3.2) is implied by the first equation, which may be proved by induction using the properties of $\varphi$.

The following proposition shows how $M_{\mathrm{w}}, \lambda_{\mathrm{w}}$, and $\lambda_{\mathrm{s}}$ (or, equivalently, $M_{\mathrm{s}}$ ) are affected by the action of a local isomorphism.

Proposition 3.1. Let $(X, E(X))$ and $(Y, E(Y))$ be two connected multigraphs, and suppose that there exists a local isomorphism $\varphi$ from $X$ onto $Y$. The following assertions hold.

(a) $\lambda_{\mathrm{W}}(X)=\lambda_{\mathrm{W}}(Y)$.

(b) $\lambda_{\mathrm{s}}(X) \geq \lambda_{\mathrm{s}}(Y)$. If there exists $y \in Y$ such that $\left|\varphi^{-1}(y)\right|<+\infty$ then $\lambda_{\mathrm{s}}(X)=\lambda_{\mathrm{s}}(Y)$.

(c) $M_{\mathrm{W}}(X)=M_{\mathrm{W}}(Y)$. Moreover, $Y$ satisfies condition (3.1) if and only if $X$ satisfies it.

Proof. (a) Let $\eta_{t}$ be a $\lambda$-BRW process on $X$ starting with one particle at site $x$. We may easily show that

$$
\xi_{t}(y):=\sum_{x \in \varphi^{-1}(y)} \eta_{t}(x)
$$

is a $\lambda$-BRW process on $Y$ starting with one particle at site $\varphi(x)$. It is clear that $\eta_{t}$ survives globally if and only if $\xi_{t}$ does; this implies that $\lambda_{\mathrm{W}}(X)=\lambda_{\mathrm{W}}(Y)$. 
(b) If $\eta_{t}$ survives locally then $\xi_{t}$ does; hence, $\lambda_{\mathrm{s}}(X) \geq \lambda_{\mathrm{s}}(Y)$. On the other hand, given that $\left|\varphi^{-1}(y)\right|<+\infty$, if we start the process $\eta_{t}$ with one particle at a site $x \in \varphi^{-1}(y)$ and $\xi_{t}$ survives locally (in $y$ ), the same must be true for $\eta_{t}$ at some $z \in \varphi^{-1}(y)$ and, hence, at $x \in X$.

(c) It is a simple consequence of the equality $T_{x}^{n}(X)=T_{\varphi(x)}^{n}(Y)$ which holds for all $x \in X$ and $n \in \mathbb{N}$.

According to the previous proposition, if $(X, E(X))$ is locally isomorphic to a multigraph $(Y, E(Y))$ which satisfies the hypotheses of Theorem 3.3, then the same conclusions of this theorem hold for $(X, E(X))$. In particular, if $(Y, E(Y))$ is a finite multigraph then $M_{\mathrm{w}}(Y)=$ $M_{\mathrm{s}}(Y)$ and $\lambda_{\mathrm{w}}(Y)=\lambda_{\mathrm{s}}(Y)$.

We note that both quasi-transitive graphs and regular graphs are $\mathcal{F}$-multigraphs. Indeed, if $X$ is a quasi-transitive graph, we take $Y$ as the quotient space with respect to the action of the automorphism group, $\varphi$ as the quotient map, and $n_{y y^{\prime}}:=\left|\varphi^{-1}\left(y^{\prime} \cap D_{X}(x)\right)\right|$, where $\varphi(x)=y$ (this definition does not depend on the choice of $x$ ); regular graphs of degree $k$ may be mapped on the one-point multigraph with $k$ loops (and $M_{\mathrm{w}}=k$ ). Nevertheless, this class contains graphs which are neither regular nor quasi-transitive (see Examples 3.1, 3.2, and 3.3, below). Moreover, the 'regularity' of $\mathcal{F}$-multigraphs is only 'local'; indeed, we can easily construct examples of quite irregular $\mathcal{F}$-graphs.

The following lemma gives a sufficient condition for a graph to be an $\mathcal{F}$-graph.

Lemma 3.2. Let us consider a graph $(X, E(X))$ such that, for all $x, y \in X$ with $\operatorname{deg}(x)=$ $\operatorname{deg}(y)$, we have

$$
|\{z \in D(x): \operatorname{deg}(z)=j\}|=|\{z \in D(y): \operatorname{deg}(z)=j\}| \text { for all } j=1, \ldots, M .
$$

Then $(X, E(X))$ is an $\mathcal{F}$-graph.

Proof. Take

$Y:=\{i \in \mathbb{N}:$ there exists $x \in X, \operatorname{deg}(x)=i\} \quad$ and $\quad n_{i j}:=|\{z \in D(x): \operatorname{deg}(z)=j\}|$

for some $x \in X$ such that $\operatorname{deg}(x)=i$ (the definition does not depend on $x$ ) and $\varphi:=\operatorname{deg}$.

In analogy to Theorem 3.3, the following theorem describes another class of multigraphs satisfying condition (3.1).

Theorem 3.4. If $(X, E(X))$ is an $\mathcal{F}$-multigraph then it satisfies condition (3.1) and $\lambda_{\mathrm{w}}=$ $1 / M_{\mathrm{w}}$.

Proof. Let $(X, E(X))$ be locally isomorphic to the finite multigraph $(Y, E(Y))$. We note that, being finite, $Y$ satisfies condition (3.1), whence, by Proposition 3.1, $X$ also does.

Remark 3.2. It is natural to wonder how $M_{\mathrm{s}}, M_{\mathrm{w}}, \lambda_{\mathrm{s}}$, and $\lambda_{\mathrm{w}}$ are affected by local modifications of the multigraphs $(X, E(X))$ (such as, for instance, attaching a complete, finite graph to a vertex of $X$ or removing a set of vertices and/or edges).

If $(X, E(X))$ and $(Y, E(Y))$ are two multigraphs and $\psi: Y \rightarrow X$ is an injective map such that $n_{\psi(x) \psi(y)} \geq \tilde{n}_{x y}$ for all $x, y \in Y$ (where $\tilde{n}$ refers to $Y$ ) then $\lambda_{\mathrm{W}}(X) \leq \lambda_{\mathrm{W}}(Y), \lambda_{\mathrm{s}}(X) \leq \lambda_{\mathrm{s}}(Y)$, $M_{\mathrm{W}}(X) \geq M_{\mathrm{w}}(Y)$, and $M_{\mathrm{S}}(X) \geq M_{\mathrm{S}}(Y)$.

In certain cases it is easy to show that the existence of a pure weak phase on $X$ implies the existence of a pure weak phase on some submultigraph; indeed, if $Y$ is a finite subset of $X$ such that $X \backslash Y$ is divided into a finite number of connected multigraphs $X_{1}, \ldots, X_{n}$ 
(which is certainly true if $n_{x y}>0$ is equivalent to $n_{y x}>0$ for all $x, y \in X \backslash Y$ ) then, for every $\lambda \in\left(\lambda_{\mathrm{w}}(X), \lambda_{\mathrm{s}}(X)\right)$, the $\lambda$-BRW leaves eventually almost surely the subset $Y$. Hence, it survives (globally but not locally) at least on one connected component; this means that, although $\lambda_{\mathrm{S}}\left(X_{i}\right) \geq \lambda_{\mathrm{s}}(X)$ and $\lambda_{\mathrm{W}}\left(X_{i}\right) \geq \lambda_{\mathrm{W}}(X)$ for all $i=1, \ldots, n$, there exists $i_{0}$ such that $\lambda_{\mathrm{W}}\left(X_{i_{0}}\right)=\lambda_{\mathrm{W}}(X)$. The existence of a pure weak phase on $X_{i_{0}}$ follows from $\lambda_{\mathrm{S}}\left(X_{i_{0}}\right) \geq$ $\lambda_{\mathrm{s}}(X)>\lambda_{\mathrm{w}}(X)=\lambda_{\mathrm{w}}\left(X_{i_{0}}\right)$.

Moreover, if there exists a subset $Y$ as above such that $\lambda_{\mathrm{W}}\left(X_{i}\right)>\lambda_{\mathrm{W}}(X)$ for all $i$ then there is no pure weak phase for the BRW on $X$. Take, for instance, a graph $\left(X^{\prime}, E\left(X^{\prime}\right)\right)$ and $k \in \mathbb{N}$ such that $1 / k<\lambda_{\mathrm{W}}\left(X^{\prime}\right)$. Attach a complete graph of degree $k$ to a vertex of $X^{\prime}$. We obtain a new graph $X$ such that $\lambda_{\mathrm{s}}(X)=\lambda_{\mathrm{W}}(X) \leq 1 / k<\lambda_{\mathrm{W}}\left(X^{\prime}\right)$; hence, even if the BRW on $X^{\prime}$ has a pure weak phase, the BRW on $X$ has none.

\subsection{Critical behaviors}

It is well known that any critical branching process dies out almost surely. In Theorem 3.1 we associated to the BRW a (discrete-time) branching process in such a way that the progeny of the BRW in a fixed site $x$ coincides with the total progeny of the branching process. This allows us to study the strong critical behavior of the BRW (and the weak critical behavior for a BRW on an $\mathcal{F}$-multigraph).

Theorem 3.5. For each multigraph $(X, E(X))$, if $\lambda=\lambda_{\mathrm{s}}$ then the $\lambda$-BRW dies out locally almost surely. For each $\mathcal{F}$-multigraph, if $\lambda=\lambda_{\mathrm{w}}$ then the $\lambda$-BRW on $X$ dies out globally almost surely.

Proof. Recall that (see the proof of Theorem 3.1) the $\lambda$-BRW survives if and only if the branching process with an expected number of children $\Phi(o, o \mid \lambda)$ does. Since

$$
\Phi\left(o, o \mid \frac{1}{M_{\mathrm{S}}}\right) \leq 1 \quad \text { and } \quad \lambda_{\mathrm{s}}=\frac{1}{M_{\mathrm{S}}},
$$

there is an almost sure local extinction at $\lambda_{\mathrm{s}}$.

Now suppose that $X$ is locally isomorphic to the finite multigraph $Y$. Since the global behavior of the $\lambda$-BRW $\eta_{t}$ on $X$ is the same as the corresponding behavior of the induced $\lambda$-BRW $\xi_{t}$ on $Y$ (see the proof of Proposition 3.1), then Theorem 3.2 and Proposition 3.1 imply that $\lambda_{\mathrm{W}}(X)=1 / M_{\mathrm{W}}(X)=\lambda_{\mathrm{W}}(Y)=\lambda_{\mathrm{s}}(Y)$. But we just proved that each $\lambda_{\mathrm{s}}$-BRW dies out locally almost surely; moreover, since $Y$ is finite, $\xi_{t}$ dies out globally almost surely; hence, the same holds for $\eta_{t}$.

\subsection{Nonamenability and weak phase}

In this subsection we consider only nonoriented multigraphs. A multigraph $(X, E(X))$ is nonamenable if

$$
\inf \left\{\frac{\left|\partial_{E}(S)\right|}{|S|}: S \subseteq X,|S|<\infty\right\}=: \iota_{X}>0,
$$

where $\partial_{E}(S)$ is the set of edges $(x, y, i) \in E(X)$ such that $x \in S$ and $y \notin S$.

We define $N: l^{2}(X) \rightarrow l^{2}(X)$ by $N f(x):=\sum_{y \in X} n_{x y} f(y)$, which is a bounded, linear operator with $\|N\| \leq M$. It is well known that, on a regular, nonoriented graph (where $M=$ $M_{\mathrm{w}}$ ), the existence of the weak phase is equivalent to nonamenability (see [15, Theorem 2.4]). Indeed, on regular, nonoriented graphs, $M_{\mathrm{S}}<M_{\mathrm{W}}$ is equivalent to nonamenability: we can easily prove that $M_{\mathrm{s}}=\|N\|$ (see Lemma 3.3, below, and the reference therein); moreover, $\|N\|=M\|P\|$, where $P$ is the transition operator associated to the simple random walk and 
Gerl [2] proved that $\|P\|<1$ is equivalent to nonamenability. Hence, using Theorem 3.1 and Theorem 3.4, we obtain an alternative proof of [15, Theorem 2.4].

Now we show that, for nonoriented $\mathcal{F}$-multigraphs, nonamenability is equivalent to the existence of a pure weak phase which, in turn, is equivalent to $M_{\mathrm{S}}<M_{\mathrm{W}}$.

Lemma 3.3. The operator $N$ is self-adjoint and $\|N\|=\rho(N)=M_{\mathrm{s}}$, where $\rho(N)=$ $\lim _{n \rightarrow \infty}\left\|N^{n}\right\|^{1 / n}$ is the spectral radius of $N$.

Proof. The self-adjointness of $N$ is easy and $\|N\|=\rho(N)$ is a standard property which follows from the spectral theorem for any normal (hence self-adjoint) operator. To prove that $M_{\mathrm{S}}=\|N\|$, we proceed essentially as in [15, Lemma 2.2].

The following theorem implies the analogous results for regular and quasi-transitive graphs.

Theorem 3.6. Let $(X, E(X))$ be a nonoriented $\mathcal{F}$-multigraph. Then $\lambda_{\mathrm{w}}<\lambda_{\mathrm{s}}$ if and only if $(X, E(X))$ is nonamenable.

Before proving this statement, we need a technical result concerning the Dirichlet norm of $l^{2}$ functions. Given $f \in l^{2}(X)$, define

$$
\|f\|_{D(2)}=\left(\sum_{x, y \in X} n_{x y}|f(x)-f(y)|^{2}\right)^{1 / 2} .
$$

Lemma 3.4. Let $(X, E(X))$ be a nonamenable multigraph. Then there exists $c>0$ such that, for all $f \in l^{2}(X)$,

$$
\|f\|_{D(2)} \geq c\|f\|_{2} .
$$

Proof. The proof is analogous to the one of [15, Theorem 2.6] (we have to deal carefully with the presence of $n_{x y}$ ); hence, we omit it.

Proof of Theorem 3.6. We follow the proof of [15, Theorem 3.1]. Let $(X, E(X))$ be nonamenable, let $N=\left(n_{x y}\right)_{x, y \in X}$ be its adjacency matrix, and let $\tilde{N}=\left(\tilde{n}_{x y}\right)_{x, y \in Y}$ be the adjacency matrix of the finite multigraph $(Y, E(Y))$, which $(X, E(X))$ is locally isomorphic to. We must prove that $\|N\|<M_{\mathrm{w}}$. By definition of local isomorphism, we have $\tilde{n}_{\varphi(x) \varphi(y)}=$ $\sum_{z: \varphi(z)=\varphi(y)} n_{x z}$. By the Perron-Frobenius theorem, $\tilde{N}$ has largest positive eigenvalue $M_{\mathrm{W}}$ with associated positive eigenvector $\left(a_{1}, \ldots, a_{k}\right)$ ( $k$ being the cardinality of $Y$ ). Then

$$
M_{\mathrm{w}} a_{\varphi(x)}=\sum_{y^{\prime} \in Y} \tilde{n}_{\varphi(x) y^{\prime}} a_{y^{\prime}}=\sum_{y^{\prime} \in Y} \sum_{y \in \varphi^{-1}\left(y^{\prime}\right)} n_{x y} a_{\varphi(y)}=\sum_{y \in X} n_{x y} a_{\varphi(y)} .
$$

Take $f \in l^{2}(X)$. Applying (3.3) and the fact that $(X, E(X))$ is nonoriented,

$$
M_{\mathrm{w}}^{2}\|f\|_{2}^{2}=M_{\mathrm{w}}^{2} \sum_{y \in X}(f(y))^{2}=\sum_{x \in X}\left(\sum_{z \in X} n_{x z} a_{\varphi(z)}\right)\left(\sum_{y \in X} n_{x y} \frac{(f(y))^{2}}{a_{\varphi(y)}}\right) .
$$

Hence,

$$
M_{\mathrm{w}}^{2}\|f\|_{2}^{2}-\|N f\|_{2}^{2}=\sum_{x \in X} \sum_{z, y \in X} n_{x z} n_{x y}\left(\frac{a_{\varphi(z)}}{a_{\varphi(y)}}(f(y))^{2}-f(z) f(y)\right) \geq \frac{1}{2}\left(\min a_{i}\right)^{2}\|g\|_{D(2)},
$$

where $g(x)=f(x) / a_{\varphi(x)}$ is considered as a map on the multigraph $G^{2}=(X, \bar{E}(X))$ with adjacency matrix $\bar{N}$ defined by $\bar{n}_{x y}=\sum_{z \in X} n_{z x} n_{z y}$ and $\bar{E}(X):=\{(y, z, i): 1 \leq i \leq$ $\left.\bar{n}_{x y}\right\}$. Applying Lemma 3.4 to each connected component of $G^{2}$ (note that each of them is 
nonamenable) and noting that $\|g\|_{2}^{2} \geq D^{2}\|f\|_{2}^{2}$ for $D^{-1}=\max \left(a_{i}\right)$, we have, for some $C>0$,

$$
M_{\mathrm{w}}^{2}\|f\|_{2}^{2}-\|N f\|_{2}^{2} \geq C\|f\|_{2}^{2},
$$

whence $\|N\| \leq \sqrt{M_{\mathrm{w}}^{2}-C}<M_{\mathrm{w}}$.

Now suppose that $(X, E(X))$ is amenable and fix $\varepsilon>0$. Then, for some finite set $S \subset X$, $\left|\partial_{E} S\right| /|S|<\varepsilon$. Define $f(x)=a_{\varphi(x)} \mathbf{1}_{S}(x)$. If $x \in S$ and $D(x) \cap S^{\mathrm{c}}=\varnothing$ then, by (3.3), $N f(x)=M_{\mathrm{w}} f(x)$. Hence,

$$
\|N f\|_{2}^{2} \geq M_{\mathrm{w}}^{2}\|f\|_{2}^{2}-2 M_{\mathrm{w}} \varepsilon|S|\left(\max a_{i}\right)^{2}
$$

and

$$
\frac{\|N f\|_{2}^{2}}{\|f\|_{2}^{2}} \geq M_{\mathrm{w}}^{2}-2 \varepsilon M_{\mathrm{w}}\left(\frac{\max a_{i}}{\min a_{i}}\right)^{2} .
$$

By taking $\varepsilon$ arbitrarily small, we prove that $\|N\| \geq M_{\mathrm{W}}$, whence $M_{\mathrm{S}}=M_{\mathrm{W}}$ (recall that $\left.\|N\|=M_{\mathrm{s}} \leq M_{\mathrm{W}}\right)$.

\subsection{Examples}

The first two explicit examples listed hereafter show that the class of $\mathcal{F}$-multigraphs is larger than the union of regular and quasi-transitive multigraphs. Both these examples are modifications of regular graphs: Example 3.1 is obtained by attaching an edge to each vertex and Example 3.2 is obtained by drawing a 'bridge with intermediate station' between some of the vertices.

Example 3.1. Take a square and attach to every vertex a branch of a homogeneous tree of degree 3 , obtaining a regular graph (of degree 3 ) which is not quasi-transitive. If we now attach to each vertex a new edge with a new endpoint, we obtain a nonoriented, nonamenable $\mathcal{F}$-graph $(X, E(X))$ which is neither regular nor quasi-transitive. It is easily seen (by Lemma 3.2) to be locally isomorphic to a multigraph with adjacency matrix

$$
N=\left(\begin{array}{ll}
3 & 1 \\
1 & 0
\end{array}\right)
$$

According to Theorem 3.6, the BRW on this graph has a pure weak phase.

Example 3.2. Take an infinite graph $(X, E(X))$ with a set of vertices $X=\left\{x_{1}, x_{2}, \ldots\right\}$. If $Y=\left\{y_{1}, y_{2}, \ldots\right\}$ is another countable set, disjoint from $X$, we may consider the graph with a set of vertices $Z:=X \cup Y$ and

$$
E(Z):=E(X) \cup \bigcup_{i=1}^{\infty}\left\{\left(x_{2 i-1}, y_{i}\right),\left(y_{i}, x_{2 i-1}\right),\left(x_{2 i}, y_{i}\right),\left(y_{i}, x_{2 i}\right)\right\} ;
$$

roughly speaking, we join $x_{2 i-1}$ and $x_{2 i}$ by a bridge and we cut this bridge into two edges by using a new vertex $y_{i}$. If the graph $X$ is nonamenable then it is possible to show that the (multi)graph $Z$ is nonamenable as well. By choosing $(X, E(X))$ regular (with deg $\equiv k$ ), we obtain an $\mathcal{F}$-graph which (by Lemma 3.2) is locally isomorphic to a multigraph with adjacency matrix

$$
N=\left(\begin{array}{ll}
k & 1 \\
2 & 0
\end{array}\right)
$$

Again, by choosing accurately $(X, E(X))$ and ordering its vertices wisely, we may obtain a graph which is neither quasi-transitive nor regular. 
The following trees are natural examples of graphs which are not quasi-transitive and, nevertheless, are not 'too irregular'. We show that, for these trees, nonamenability is equivalent to the existence of a pure weak phase and the proof is not a direct application of Theorem 3.6.

Example 3.3. Given a sequence of positive natural numbers $\left\{m_{k}\right\}_{k \geq 1}$, we construct a nonoriented, rooted tree $\mathcal{T}$ (with root $o$ ) such that if $x \in \mathcal{T}$ satisfies $\rho(o, x)=k$ then it has $m_{k+1}$ neighbors $y$ such that $\rho(0, y)=k+1$. We call this radial graph the $T_{\left\{m_{k}\right\}}$-tree. If the sequence is periodical of period $d$ then Theorem 3.3 applies with $x_{0}=o, n_{0}=d$, and $Y:=\cup_{n \in \mathbb{N}} B(o, n d)$, and $\varphi_{y}$ (where $y \in Y$ ) maps isomorphically the tree $\mathcal{T}$ onto the subtree branching from $y$. We call $T_{i}$ the $T_{\left\{m_{k}^{\prime}\right\}}$-tree obtained by means of this construction, where $m_{k}^{\prime}:=m_{k+i-1}$. Roughly speaking, we construct $T_{1}, \ldots, T_{d}$ by using cyclic permutations of the sequence $\left\{m_{1}, \ldots, m_{d}\right\}$. Obviously, $\mathcal{T}=T_{1}$. Since $T_{i}$ may be mapped into $T_{j}$ for all $i, j=1, \ldots, d$ (in the sense of Remark 3.2), $\lambda_{\mathrm{w}}\left(T_{i}\right), \lambda_{\mathrm{s}}\left(T_{i}\right), M_{\mathrm{W}}\left(T_{i}\right)$, and $M_{\mathrm{S}}\left(T_{i}\right)$ do not depend on $i$.

Let us consider the finite cyclic graph $\tilde{Y}:=\left\{y_{1}, \ldots, y_{d}\right\}$, where $n_{y_{i} y_{i+1}}=n_{y_{i+1} y_{i}}=1$ for all $i=1, \ldots d$ (with the identification $y_{d+1}=y_{1}$ ). To each vertex $y_{i}$ we attach $m_{i}-1$ copies of $T_{i+1}$ (again with the identification $T_{d+1}=T_{1}$ ) by using $m_{i}-1$ distinct two-way edges. We denote this connected, nonoriented $\mathcal{F}$-graph by $(X, E(X))$; indeed, it may be mapped onto the finite multigraph $Y^{\prime}$, where $Y^{\prime}=\tilde{Y}$, and $n_{y_{i} y_{i+1}}^{\prime}=m_{i}$ and $n_{y_{i+1} y_{i}}^{\prime}=1$ for all $i=1, \ldots d$. Note that $X$ is neither quasi-transitive nor regular, unless $m_{i}=1$ for all $i$. The graph $X$ is nonamenable if and only if $T_{1}$ is nonamenable, that is, if and only if there exists $i$ such that $m_{i} \geq 2$. In this case, according to Theorem 3.6, $\lambda_{\mathrm{w}}(X)<\lambda_{\mathrm{s}}(X)$; hence, by Remark 3.2 (considering $X \backslash \tilde{Y}$ ), there exists $i$ such that $\lambda_{\mathrm{W}}\left(T_{i}\right)<\lambda_{\mathrm{s}}\left(T_{i}\right)$. This means that, for all $i$, we have $\lambda_{\mathrm{w}}\left(T_{i}\right)<\lambda_{\mathrm{s}}\left(T_{i}\right)$ and there is a pure weak phase on $T_{i}$. On the other hand, if $m_{i} \equiv 1$ for all $i=1, \ldots, d$ then there is no pure weak phase (Remark 3.1).

\section{Modified BRW and BRW on weighted graphs}

\subsection{Modified BRW}

In this subsection we consider an irreducible random walk $(X, P)$. In the case of simple random walks some of the results of this subsection may also be found in [15]. We study the modified BRW where each particle at site $x$ dies at rate 1 and breeds at rate $\lambda$ and it sends the offspring randomly according to the probability distribution $p(x, \cdot)$.

We denote by $p^{(n)}(x, y)$ the $n$-step transition probabilities from $x$ to $y(n \geq 0)$ and by $f^{(n)}(x, y)$ the probability that the random walk starting from $x$ hits $y$ for the first time after $n$ steps $(n \geq 1)$. Then we define the corresponding generating functions $G(x, y \mid z)=$ $\sum_{n \geq 0} p^{(n)}(x, y) z^{n}$ and $F(x, y \mid z)=\sum_{n \geq 1} f^{(n)}(x, y) z^{n}$, where $x \in X$ and $z \in \mathbb{C}$ (further details can be found in [16, Chapter I.1.B], where $F$ is called $U$ ).

The expected number of trails along a path $\Pi=\left\{x_{0}, \ldots, x_{n}\right\}$ is equal to $\lambda^{n} \prod_{i=0}^{n-1} p\left(x_{i}, x_{i+1}\right)$. Hence, the expected number of trails along paths starting from $x$ and reaching $y$ for the first time is equal to $F(x, y \mid \lambda)$. If $x$ is equal to $y$, we call them first generation trails in $x$. Since $G(x, x \mid \lambda)=1 /(1-F(x, x \mid \lambda))$ and the radius $R$ of $G$ does not depend on the choice of $x$, we have $R=\max \{\lambda: F(x, x \mid \lambda) \leq 1\}$.

Theorem 4.1. For the modified $B R W, \lambda_{\mathrm{w}}=1$ and, if $\lambda=1$, there is global extinction almost surely. Moreover, $\lambda_{\mathrm{s}}=R$ and, if $\lambda=R$, there is local extinction almost surely.

Proof. The total number of particles $T_{t}$ is a branching process with rate $\lambda$, whence the claim for $\lambda_{\mathrm{w}}$ follows. As for the second claim, the proof is the same as in Theorems 3.1 and 3.5 using $F$ instead of $\Phi$. 
The following corollary is the analog of Theorem 3.6 (see [2] for the definition of a strongly reversible random walk).

Corollary 4.1. For the modified BRW, the existence of a pure weak phase is equivalent to $R>1$. If $P$ is a strongly reversible random walk then the existence of the pure weak phase is equivalent to nonamenability.

Proof. The result is a simple consequence of Theorem 4.1 and the main theorem of [2].

\subsection{BRW on weighted graphs}

Our methods apply, with minor modifications, to more general BRWs, which generalize simultaneously BRWs on multigraphs and modified BRWs.

Let us consider $(X, N)$, where $X$ is a countable (or finite) set and $N=\left(n_{x y}\right)_{x, y \in X}$ is a matrix of nonnegative weights (that is, $n_{x y} \geq 0$ ) such that $\sup _{x \in X} \sum_{y \in X} n_{x y}=M<\infty$. We suppose that $N$ is irreducible in the sense that $(X, E(X))$, where $E(X):=\left\{(x, y) \in X \times X: n_{x y}>0\right\}$ is a connected graph. We call $(X, N)$ a weighted graph.

The $\lambda$-BRW is defined by setting the reproduction rate on every edge $(x, y)$ as $\lambda n_{x y}$; hence, to each path $\left\{x_{0}, \ldots, x_{n}\right\}$ there corresponds a weight $\prod_{i=0}^{n-1} n_{x_{i} x_{i+1}}$. We define $\gamma_{x, y}^{n}, T_{x}^{n}, \phi_{x, y}^{n}$, $M_{\mathrm{S}}$, and $M_{\mathrm{w}}$ as in Subsection 2.1.

It is clear that the BRW on multigraphs and the modified (according to an irreducible random walk) BRW may be viewed as BRWs on weighted graphs. Moreover, the expected number of trails along a path $\left\{x_{0}, x_{1}, \ldots, x_{n}\right\}$ is $\lambda^{n} \prod_{i=0}^{n-1} n_{x_{i} x_{i+1}}$. Substituting the word 'multigraphs' with 'weighted graphs', all the results of Sections 2 and 3 still hold (with the exception of Theorems 3.2 and 3.3) with unimportant modifications. In particular, extending Definition 3.1 verbatim to weighted graphs, we can prove Theorem 3.4, since in this case $n_{x y}$ may take just a finite number of values and it is possible to apply Lemma 2.1 as we did in Theorem 3.2. Clearly, we extend the results on the critical behaviors (Theorem 3.5) to this case. For regular weighted graphs (that is, $\sum_{y \in X} n_{x y}=M$ for all $x \in X$ ), we prove results analogous to the ones of Subsection 4.1 .

\section{Open questions}

As we stated in Section 1, this paper is motivated by three main issues: the identification of the critical value $\lambda_{\mathrm{w}}$, the behavior of the process when $\lambda=\lambda_{\mathrm{s}}$ or $\lambda=\lambda_{\mathrm{w}}$, and the existence of the pure weak phase.

To complete the first point, one should verify whether the equality $\lambda_{\mathrm{w}}=1 / M_{\mathrm{W}}$ holds for every multigraph or if $M_{\mathrm{w}}$ characterizes the critical value $\lambda_{\mathrm{w}}$ only on a restricted class of multigraphs.

As for the second one, the open question is the following: is it possible to construct a multigraph where if $\lambda=\lambda_{\mathrm{w}}$ the process does not die out globally? In particular, is it possible to find a multigraph where $\lambda_{\mathrm{s}}=\lambda_{\mathrm{w}}$ but the $\lambda_{\mathrm{w}}$-BRW does not die out globally (it certainly does locally)?

Finally, dealing with the existence of a pure weak phase, it is well known that there is no equivalence, in general, with nonamenability. We proved that this equivalence holds, for instance, for the class of nonoriented $\mathcal{F}$-multigraphs; we do not know what can be said in the case of oriented $\mathcal{F}$-multigraphs. To be precise: is there a nonamenable, oriented $\mathcal{F}$-multigraph, where the BRW has no weak phase? On the other hand, is it possible to find an amenable, oriented $\mathcal{F}$-multigraph where $\lambda_{\mathrm{s}}=\lambda_{\mathrm{w}}$ ? 


\section{References}

[1] Cassi, D. and Regina, S. (1992). Random walks on $d$-dimensional comb lattices. Modern Phys. Lett. B 6, 1397-1403.

[2] Gerl, P. (1988). Random walks on graphs with a strong isoperimetric property. J. Theoret. Prob. 1, 171-187.

[3] Häggström, O. (2000). Markov random fields and percolation on general graphs. Adv. Appl. Prob. 32, 39-66.

[4] Harris, T. E. (1963). The Theory of Branching Processes. Springer, Berlin.

[5] Hueter, I. AND Lalley, S. P. (2000). Anisotropic branching random walks on homogeneous trees. Prob. Theory Relat. Fields 116, 57-88.

[6] JAGERs, P. (1995). Branching processes as population dynamics. Bernoulli 1, 191-200.

[7] Liggett, T. M. (1996). Branching random walks and contact processes on homogeneous trees. Prob. Theory Relat. Fields 106, 495-519.

[8] Liggett, T. M. (1999). Branching random walks on finite trees. In Perplexing Problems in Probability (Progress Prob. 44), Birkhäuser, Boston, MA, pp. 315-330.

[9] Lyons, R. (1989). The Ising model and percolation on trees and tree-like graphs. Commun. Math. Phys. 125, 337-353.

[10] Lyons, R. (1990). Random walks and percolation on trees. Ann. Prob. 18, 931-958.

[11] LyOns, R. (2000). Phase transitions on nonamenable graphs. Probabilistic techniques in equilibrium and nonequilibrium statistical physics. J. Math. Phys. 41, 1099-1126.

[12] Madras, N. and Schinazi, R. (1992). Branching random walks on trees. Stoch. Proc. Appl. 42, $255-267$.

[13] Pemantle, R. (1992). The contact process on trees. Ann. Prob. 20, 2089-2116.

[14] Pemantle, R. And Stacey, A. M. (2001). The branching random walk and contact process on Galton-Watson and nonhomogeneous trees. Ann. Prob. 29, 1563-1590.

[15] Stacey, A. M. (2003). Branching random walks on quasi-transitive graphs. Combin. Prob. Comput. 12, 345-358.

[16] WoEss, W. (2000). Random Walks on Infinite Graphs and Groups (Cambridge Tracts Math. 138). Cambridge University Press. 